\title{
Residues of 862,921 of VP3 are associated with virulence in infectious bursal disease virus strain Harbin-1
}

\author{
Renmao $\mathrm{Li}^{1,2}$, Haiying Wang ${ }^{1,3}$, Guangming Huang ${ }^{1}$, Manfu Zhang ${ }^{{ }^{*}}$ \\ ${ }^{1}$ Lab for Animal Molecular Virology, College of Biological Sciences, China Agricultural University, Beijing, China; *Corresponding \\ Author: Manfuzhang@yahoo.com \\ ${ }^{2}$ Life Sciences and Technology School, Zhanjiang Normal University, Zhanjiang, China \\ ${ }^{3}$ Department of Biochemistry and Molecular Biology, Peking University Health Center, Beijing, China
}

Received 25 February 2010; revised 28 April 2010; accepted 6 May 2010.

\begin{abstract}
Reverse genetics was used to study the effect of particular amino acids of infectious bursal disease virus (IBDV) on virulence. Using site-directed mutagenesis, altering of two amino acids in VP2 (Q253H, A284T) and VP3 (H783Q, V862M, I921V) in the segment $A$ of a Chinese very virulent IBDV field strain Harbin-1, 4 virus mutants including $\mathrm{H}_{253 / 284}, \mathrm{H}_{783 / 862}, \mathrm{H}_{862 / 921}, \mathrm{H}_{921 / 783}$ were rescued. To evaluate the characteristics of the recovered viruses in vivo, we inoculated 4-week-old chickens with virus mutants and rescued Harbin1 (rHarbin-1), analyzed their bursae for pathological lesions 4 days postinfection. rHarbin-1 and $H_{783 / 862}, H_{253 / 284}$ caused severe bursal lesion, milder lesion for $\mathrm{H}_{862 / 921}$, mildest for $\mathrm{H}_{921 / 783}$. However, $\mathrm{H}_{253 / 284}$ caused the lowest mortality. The results showed that residue at position Q253, A284 of VP2 and V862, 1921 of VP3 gene are involved with virulence, but there is difference between VP2 and VP3's role in virulence. The ability of 862 and 921 to control virulence in VP3 is stronger than 253 and 284.
\end{abstract}

Keywords: IBDV; VP3; Mutagenesis; Reverse Genetics; Virulence

\section{INTRODUCTION}

Infectious bursal disease (IBD) is a highly contagious disease among young chickens and characterized by the destruction of the bursa of Fabricius. IBD was first described by Cosgrove [1], but in China the first case was

*Part of the contents in this article was presented in Shanghai University in June of 2009 reported in 1979 [2]. Nowadays IBD has spread worldwide and continues to threat the poultry industry. Infectious bursal disease virus (IBDV) is the causative agent of the disease, belonging to Avibirnavirus genus of the Birnaviridae family [3]. Europe had experienced the emergence of very virulent infectious bursal disease virus (vvIBDV) which can cause up to $70 \%$ flock mortality $[4,5]$. Meanwhile, vvIBDV infections also have been observed in Asia and in South America [6].

The genome of IBDV consists of two segments of double-stranded RNA (dsRNA), approximately $3.4 \mathrm{~kb}$ (segment A) and $2.7 \mathrm{~kb}$ (segment B) in length [7]. Segment A contains two partially overlapping open reading frames (ORFs). The larger ORF encodes a polyprotein (1,012 amino acids, $110 \mathrm{kDa})$ that is autocatalytically cleaved to yield the viral proteins pVP2 (VPX) (48 kDa), VP4 (29 kDa) and VP3 (33 kDa). During virus maturation, pVP2 is processed into matured VP2 (41 to 38 $\mathrm{kDa}$ ), probably resulting from site-specific cleavage of pVP2 by a host cell-encoded protease [8]. The smaller ORF encodes the nonstructural protein VP5 (145 to 149 amino acids, $17 \mathrm{kDa}$ ). Segment B encodes VP1 (970 $\mathrm{kDa}$ ) having putative RNA-dependent RNA polymerase activity $[9,10]$. This protein is covalently linked to the $5^{\prime}$ ends of the genomic RNA segments or present at a free form $[11,12]$. VP2 and VP3 are the major structural protein of the virion. The VP2 is the major host-protective antigen of IBDV and contains the determinants responsible for causing antigenic variation [13-15]. Position 279 and 284 amino acids in the VP2 variable region possibly contribute to virulence of IBDV [16]. Residues 253 and 284 of the VP2 protein of the variant virus are necessary for tissue culture infectivity [17]. The virulence and pathogenic-phenotype markers of IBDV reside in VP2 and residues at position 253 (Gln), 279 (Asp) and 284 (Ala) of VP2 are involved in the virulence and 
pathogenic phenotype of virulent IBDV [18-20]. However, recent study demonstrated VP2 is not the sole determinant of the very virulent phenotype [21]. C-terminal part of VP3 may play a decisive role in controlling the virulence [22]. VP3 could play an important role in receptor-mediated virus-cell attachment, which implied that VP3 has relation with virulence [23].

In order to verify if VP3 have molecular determinant of virulence for Chinese vvIBDV strain Harbin-1, amino acids in VP3 among Harbin-1, D78 (vaccine strain), TY89 (IBDV serotype II) were aligned, the different amino acids among them were listed in Table 1. TY89 could not infect B lymphocytes, having no virulence to B lymphocytes, and D78 has mild virulence to B lymphocytes. Based on the result of alignment the amino acids in VP3 that maybe involved in virulence could be found. Position 783 and 862 in Harbin-1 have different amino acids from D78, however, position 921 is different from TY89. To prove their role in virulence, position 783, 862 and 921 in VP3 were mutated subsequently to obtain the combination of two points mutation. As a control, position 253 and 284 in VP2 hypervarible region was mutated at the same time. By use of cRNA-based reverse-genetics system for IBDV [20], four virus mutants were recovered. Furthermore, the characteristics of recovered virus in vitro and in vivo were described and the amino acids responsible for virulence. In this paper we report the discovery that residues of 783 , 862,921 of VP3 are associated with virulence of IBDV.

\section{MATERIALS AND METHODS}

\subsection{Virus and Cells}

The very virulent strain Harbin-1 was kindly given by Harbin Veterinary Research Institute of the Chinese Academy of Agricultural Sciences. Harbin-1 causes 100\% morbidity and mortality of specific-pathogen-free (SPF) chickens, the mean infection lethal dose $\left(\mathrm{ILD}_{50}\right)$ for SPF embryo is $10^{-4} / 0.2 \mathrm{ml}$. Primary bursal cells were derived from 18-day-old embryonated SPF eggs (Merial, Beijing, China) and were grown in Dulbecco's minimal essential medium (DMEM, Sigma Aldrich, St. Louis, MO, USA) supplemented with $10 \%$ fetal calf serum (FCS) and maintained with DMEM with 5\% FBS [22]. Transfection experiments were performed on primary bursal cells. All virus mutants including $\mathrm{H}_{253 / 284}, \mathrm{H}_{783 / 862}, \mathrm{H}_{862 / 921}$, $\mathrm{H}_{921 / 783}$ and rHarbin-1, Harbin-1 were used as the viruses for challenge at a dose of 1200 pfu per animal via eye and nose drop.

\subsection{Construction of Full-Length CDNA Clones}

Several clones for segment A and segment B of Harbin1 were constructed, pGEM-T-HA (coding sequence of segment A clone), pGEM-T-H5'-A (5' non-coding sequence of segment A clone), and pGEM-T-H3'A (3' non-coding sequence of segment A clone), pGEM-T-HB (coding sequence of segment B clone), pGEM-T-H5'B ( $5^{\prime}$ non-coding sequence of segment $\mathrm{B}$ clone), $\mathrm{pG}$ EM-T-H3'B (3' non-coding sequence of segment B clone). All recombinant plasmids were based on pGEMT (Promega, Madison, WI, USA). There is partly overlapped area between $\mathrm{CR}$ (coding region) clone and NCR (non-coding region) clone for segment $\mathrm{A}$ and $\mathrm{B}$. But the overlapped area lack appropriate restriction site, thus fusion PCR was used to ligate the NCR and CR to obtain the full length cDNA clone for segment A and B. Oligonucleotides HACR1, HACR2, HANCR1, HAN CR2, HBCR1, HBCR2, HBNCR1, HBNCR2 (Table 2) were adopted for segment A and B. For transcription in vitro, EcoRI site and $\mathrm{T} 7$ promoter was introduced into $5^{\prime}$ end of oligonucleotides; XbaI site at $3^{\prime}$ end in segment A and Xhol site at $3^{\prime}$ end in segment B. The fusion PCR product of segment $\mathrm{A}$ and $\mathrm{B}$ was ligated into the T-vector (Takara Bio, Dalian, China) to obtain full-length cDNA clone named as pRHA and pRHB respectively. The sequence of final products was determined by Takara Bio Ltd.

\subsection{Site-Directed Mutagenesis}

Mutations were introduced into the cDNA of segment $\mathrm{A}$ of Harbin-1 according to the manufacture's instruction of QuickChange site-directed mutagenesis kit (Stratagene, La Jolla, CA, USA) with minor modification. Amino acid residues 253, 284, 783, 862, 921 were located in large open reading frame of segment A and their

Table 1. Different AA in VP3 for Harbin-1 and vaccine strain, serotype II strain.

\begin{tabular}{ccccccccccccccccccc}
\hline AA site & 767 & 773 & 783 & 787 & 815 & 862 & 899 & 905 & 921 & 947 & 981 & 990 & 992 & 1005 & AA site \\
\hline strain & & & & & & & & & & & & & \\
Harbin-1 & S & E & H & S & R & V & D & L & I & K & P & V & T & A & Harbin-1 \\
D78 & S & E & Q & S & R & M & D & L & I & K & L & A & T & A & D78 \\
TY89 & D & D & R & Q & K & M & E & P & V & R & P & A & S & T & TY89 \\
\hline
\end{tabular}


Table 2. Oligonucleotides used for amplification of Harbin-1 sequence*.

\begin{tabular}{|c|c|c|c|}
\hline Oligonucleotides & Qrientation & Position & Name \\
\hline СТCСТCCTTCTACAACGCTATCAT & sense & $71-94$ & HACR1 \\
\hline GAATCTAGAGGGGACCCGCGAACG XbaI & antisense & $3246-3260$ & HACR2 \\
\hline GGAATTCTAATACGACTCACTATAGGGGGGATACGATCGGTCTG & sense & $1-20$ & HANCR1 \\
\hline \multicolumn{4}{|l|}{ EcoRI } \\
\hline GATCTTGCAGGTTCGTCATCGC & antisense & $128-149$ & HANCR2 \\
\hline TGGCTACTAGGGGCGATGC & sense & $42-6$ & HBCR \\
\hline CTTCTTGAGTGGTTCCCATC & antisense & $2756-2775$ & HBCR2 \\
\hline GGAATTCTAATACGACTCACTATAGGATACGATGGGTCTGACCCT & sense & $1-21$ & HBNCR1 \\
\hline \multicolumn{4}{|l|}{ EcoRI } \\
\hline GTCACTCATGGTGGCAGAATCAT & antisense & $98-120$ & HBNCR2 \\
\hline AAACAAGCGTCCAtGGCCTTATACTGGGTGCTA & sense & $876-908$ & 253 mut 1 \\
\hline TAGCACCCAGTATAAGGCCATgGACGCTTGTTT & antisense & $876-908$ & 253 mut 2 \\
\hline GACAATGGGCTAACGACcGGCACTGACA & sense & $965-992$ & 284 mut 1 \\
\hline TGTCAGTGCCgGTCGTTAGCCCATTGTC & antisense & $965-992$ & 284 mut2 \\
\hline GACCCACTGTTCCAaTCTGCGCTCAG & sense & $2465-2490$ & 783 mut 1 \\
\hline CTGAGCGCAGAtTGGAACAGTGGGTC & antisense & $2465-2490$ & 783 mut 2 \\
\hline CTCAAAGAAGaTGGAGACTATGGG & sense & $2704-2727$ & 862 mut1 \\
\hline CCCATAGTCTCCAtCTTCTTTGAG & antisense & $2704-2727$ & 862 mut 2 \\
\hline CATCAGAAGAACAAgTCCTAAGGGCAG & sense & $2877-2903$ & 921 mut 1 \\
\hline CTGCCCTTAGGAcTTGTTCTTCTGATG & antisense & $2625-2652$ & 921 mut2 \\
\hline TAACCGTCCTCAGCTTACCC & sense & $625-644$ & outside1 \\
\hline TCAGGATTTGGGATCAGCTC & antisense & $1246-1265$ & outside2 \\
\hline CCAACCAGCGAGATAACC & sense & $1019-1036$ & inside 1 \\
\hline GGCGACCGTAACGACAG & antisense & $1212-1228$ & inside 2 \\
\hline TTCTCAGCTAATATCGATGC & sense & $842-861$ & 53,84 inupper \\
\hline GATGTGATTGGCTGGGTT & antisense & $1057-1074$ & 53,84 inlower \\
\hline GTCCAACTGGGCGACGTT & sense & $2296-2313$ & vp3 outupper \\
\hline CTGGGATTGCGATGCTTCA & antisense & $3069-3087$ & vp3 outlower \\
\hline CTTCCACCCAATGCAGGAC & sense & $2378-2396$ & 783 inupper \\
\hline CTTTGGCGACTTCGTCTATGA & antisense & 2976-2996 & 62,21 inlower \\
\hline
\end{tabular}

*Sequence and location of the oligonucleotide used in the study. Underlined nucleotides are virus-specific. Altered nucleotides for mutagenesis are in lowercase, the altered coding nucleotide triplets are highlighted in boldface. Used restriction sites are highlighted in boldface and appropriate restriction enzymes are named. The positions where the primers bind (nucleotide number) are in accordance with the sequence of strain P2 (Mundt et al., 1995).

base sites were in position $893(\mathrm{~A} \rightarrow \mathrm{T}), 984(\mathrm{G} \rightarrow \mathrm{A})$, $2483(\mathrm{~T} \rightarrow \mathrm{A}), 2718(\mathrm{G} \rightarrow \mathrm{A}), 2895(\mathrm{~A} \rightarrow \mathrm{G})$ respectively in segment of Harbin-1. First, single site-directed muta- genesis was introduced into the segment A of Harbin-1 with oligonucleotides 253 mut, 783 mut, 862 mut, 921 mut (Table 2); the mutants were sequenced to verify the 
resultant mutation; after then the second site-directed mutagenesis was introduced into the first mutation product with oligonucleotides 284 mut, 862 mut, 921 mut, 783 mut (Table 2) to obtain two point mutagenesis clone named p253/284 m, p783/862 m, p862/921 m, p921/783 m respectively. The second mutation products were sequenced by the company (Takara). The obtained muta-genized plasmids with the alteration of two amino acids, Q253H-A284T, H783Q-V862M, V862M-I921V, H783 Q-I921V were used for subsequent transcription in vitro and transfection experiments.

\subsection{Transcription and Transfection of Synthetic RNAs}

The experiment was performed by the protocol described by Mundt with minor alterations [24]. For transcription in vitro, non-mutation and mutated plasmids of segment $\mathrm{A}$ and intact segment $\mathrm{B}$ were linearized by cleavage with $\mathrm{XbaI}$ and Xholl respectively. After restrictive digestion, the products were adjusted to $0.5 \%$ SDS and incubated with proteinase $\mathrm{K}(0.5 \mathrm{mg} / \mathrm{ml})$ for 1 $\mathrm{hr}$ at $37^{\circ} \mathrm{C}$. The linearized DNA templates were recovered by ethanol precipitation, and $1 \mu \mathrm{g}$ linearized DNA was used for transcription. Segment A and segment B was transcribed respectively. Transcription reaction mixture $(30 \mu \mathrm{l})$ containing $40 \mathrm{mM}$ Tris- $\mathrm{HCl}(\mathrm{pH} 7.9), 10$ $\mathrm{mM} \mathrm{NaCl}, 6 \mathrm{mM} \mathrm{MgCl}_{2}, 2 \mathrm{mM}$ spermidine, $0.5 \mathrm{mM}$ ATP, $0.5 \mathrm{mM}$ CTP, $0.5 \mathrm{mM}$ UTP, $0.1 \mathrm{mM}$ GTP, 0.25 $\mathrm{mM}$ cap analog $\left[\mathrm{m} 7 \mathrm{G}\left(5^{\prime}\right) \mathrm{ppp}\left(5^{\prime}\right) \mathrm{G}\right]$ (Promega), 20 units RNasin, 130 units T7 RNA polymerase (Promega), and incubated at $37^{\circ} \mathrm{C}$ for $1 \mathrm{hr}$. As controls, the transcription products were treated with either DNase or RNase (Promega).

After primary bursal cells were grown to $80 \%$ confluency in 35-mm dishes, the cells were washed with DMEM (free serum) and incubated at $37^{\circ} \mathrm{C}$ for $10 \mathrm{~min}-$ utes in a $\mathrm{CO}_{2}$ incubator. The process was repeated again. Simultaneously, $60 \mu \mathrm{l}$ DMEM (free serum) was incubated with $6 \mu$ l of Lipofectin reagent (Invitrogen, Carlsbad, CA, USA) for $60 \mathrm{~min}$ in a polystyrene tube at room temperature to form Lipofectin-DMEM mixture. Synthetic RNA transcripts of both segments resuspended in $30 \mu \mathrm{l}$ of DEPC treated water were mixed and added to the DMEM-Lipofectin mixture, mixed gently and incubated on ice for $5 \mathrm{~min}$. After removing the DMEM from the monolayers in the $35-\mathrm{mm}$ dishes and replacing it with fresh $800 \mu$ of DMEM, the nucleic acid-containing mixture was added drop-wise to the cells and swirled gently. After 2 hours of incubation at $37^{\circ} \mathrm{C}$, the mixture was replaced with DMEM containing 5\% FCS (without rinsing the cells), and further incubated at $37^{\circ} \mathrm{C}$ for desired time intervals.

\subsection{Virus Recovery from cRNA and Detect the Presence of Virus by AC-ELISA, RT-PCR and Plaque Assay}

Two days after transfection, cells were frozen -thawed and centrifuged at $700 \mathrm{~g}$ to remove cellular debris. The supernatant was passaged for 4 times in the primary bursal cells, harvesting the cells for ELISA. In order to screen the recombinant virus from many samples ACELISA was performed. Each well of 96-wells polystyrene ELISA plates (Costar, Cambridge, MA, USA) were coated with $100 \mu \mathrm{l}$ of chicken polyclonal IBDV antiserum, diluted in PBS at a ratio of 1:4000. After incubation at $37^{\circ} \mathrm{C}$ for 1 hour, the plate was washed three times with washing buffer (1\% Tween 80 in PBS) and each well was blocked by $100 \mu \mathrm{l}$ of blocking buffer $(0.5 \%$ gelatin in PBS) at $37^{\circ} \mathrm{C}$ for $0.5 \mathrm{~h}$. After three washes of the plate with washing buffer, $100 \mu \mathrm{l}$ sample including positive and negative control was added in duplicate. The plate was then incubated at room temperature for $1 \mathrm{~h}$ and washed with washing buffer before $50 \mu$ of MAbs M6 or B29 [25,26]., diluted $1: 2500$ and 1:1000 in antibody diluent $(5 \% \mathrm{NaCl}$ and $4 \% \mathrm{BSA}$ in washing buffer) respectively, were added to the wells in duplicate. After incubation for $1 \mathrm{~h}$ at room temperature, the plate was washed three times with washing buffer. Subsequently, $50 \mu \mathrm{l}$ of goat anti-mouse IgG-horseradish peroxidase (Sigma) diluted 1:1000 with antibody diluent was added. One hour later at room temperature, the plate was washed three times with washing buffer. After addition $100 \mu \mathrm{TMB}$ peroxidase substrate (Kirkegaard and Perry Laboratories Inc., Gaithersburg, MD, USA) and incubated at $37^{\circ} \mathrm{C}$ for $15 \mathrm{~min}$, the reaction was stopped by adding $100 \mu \mathrm{l} 1 \mathrm{M} \mathrm{H}_{3} \mathrm{PO}_{4}$. The result was read by an ELISA reader at the optical density at $450 \mathrm{~nm}$ (OD450). If $\mathrm{OD}$ value of sample is greater than mean $\mathrm{OD}$ value plus 3 times standard deviation of negative control sample, then the sample is considered as positive and was stocked at $-86^{\circ} \mathrm{C}$ for future use.

The titre of virus mutants was determined using plaque assay [27] and prepared for future animal experiment. The titre is represented as PFU/ml.

IBDV mutants were reversely transcribed using outside 1 and nested PCR was amplified using outside 1, outside 2 , inside 1 and inside 2 primer (Table 2 ).

\subsection{Genetic Stability Analysis}

If changes in the amino acid sequence occurred during passaging viral RNA of IBDV before challenge, the identity of virus have to be confirmed. The virus mutants were subjected to RT-PCR using oligonucleotides outside 1 and outside 2 for IBDV with VP2 mutation, VP3 outupper and VP3 outlower for IBDV with VP3 muatation before challenge (Table 2). Nested PCR was ampli- 
fied with 783 inupper and 62, 21 inlower to identify virus with VP3 alteration (Table 2). Cloned PCR fragments of IBDV mutants were sequenced and obtained sequences were analyzed with DNAStar.

\subsection{Virulence of IBDV Mutants in Young SPF Chickens}

Forty eight 4-week-old SPF White Leghorn chickens were divided randomly into six groups including positive control group. Chickens were infected via eye and nose drop with total 1200 PFU. Non-inoculated hatchmates were used as negative controls. During the course of the experiment animals were observed daily for clinical signs and mortality. At 4 days p.i., all alive chickens from each group were bled and euthanized. The bursa of each chicken (include alive and dead) was removed, weighed and subdivided into two parts. One part was used for detecting the presence of IBD viral antigen by means of an AC-ELISA and RT-PCR. The second part was fixed in $10 \%$ neutral-buffered formalin for histology. Formalin-fixed bursal samples were embedded in paraffin, sectioned and stained with haematoxylin and eosin (H\&E). Microscopic bursal lesion score (BLS) was determined by histopathological analysis of the bursa. BLS was evaluated on a scale of 0 to 5 as follows: 0 , no abnormalities; 1, 1-20\%; 2, 21-40\%; 3, 41-60\%; 4, 61-80\%; and 5, 81-100\% lymphocyte depletion [28].

\subsection{Detection of Viral Antigen in Bursae after Challenge}

Bursae were homogenized with homogenizer. The presence of virus in the bursal homogenate was detected with AC-ELISA which incorporated Mab 6 recognizing VP2-located epitopes [25].

\section{RESULTS}

\subsection{Determination of Nucleotide Sequence of Harbin-1 Mutant}

To establish a reverse genetics system the complete genomic sequence of Harbin-1 mutants was determined. The mutagenized plasmids were obtained with the alteration of two amino acids, Q253H-A284T, H783QV862M, V862M-I921V, H783Q-I921V.

\subsection{Rescue of Recombinant Virus from cDNA}

Primary bursal cells were transfected with synthesized cRNA of mutated segment A and intact segment B by means of lipofectin (Invitrogen). After every transfection, the resultant supernatant was used for RT-PCR and ACELISA to detect the presence of viruses. The samples were performed to RT-PCR after IBDV antigen was de- tectable using AC-ELISA. Electrophoresis result showed that there is one $209 \mathrm{bp}$ band, whose sequence located in VP2 hypervarible region, on $1.2 \%$ agarose gel. The result of RT-PCR and AC-ELISA demonstrated that virus mutants were successfully recovered. From 10 transfection samples we obtained four mutant viruses designated as $\mathrm{H}_{253 / 284}, \mathrm{H}_{783 / 862}, \mathrm{H}_{862 / 921}, \mathrm{H}_{921 / 783}$ and rescued Harbin-1 named rHarbin-1.

\subsection{Genetic Stability Analysis}

Sequence analysis of the RT-PCR products confirmed the identity of the IBDV used. No amino acid substitutions compared to the sequence of the used plasmids (p253/284 m, p783/862 m, p861/921 m, p921/783 m) were found within the region flanked by primers used for RT-PCR, proving the genetic stability of the virus during virus pass aging.

\subsection{Virulence Determinants for VP2 and VP3 in Chinese vvIBDV Strain}

To evaluate the virulence of all virus mutants animal experiments were performed. Animals infected with vvIBDV (rHarbin-1) and $\mathrm{H}_{783 / 862}$ showed severe clinical signs of IBD. The mortality rates were $7 / 8$ for rHarbin-1, 5/8 for $\mathrm{H}_{783 / 862}, 1 / 8$ for $\mathrm{H}_{253 / 284}$ and $\mathrm{H}_{862 / 921}$. In contrast, none of the animals infected with $\mathrm{H}_{921 / 783}$ died or showed clinical signs of IBD. Bursae of chickens infected with the different virus mutants showed depletion of bursal cells in lymph nodule with remarkable differences (Figure 1). rHarbin- 1 and $\mathrm{H}_{253 / 284}, \mathrm{H}_{783 / 862}$ induced severe bursal lesion (BLS of 5, 1.6, 3.1 respectively); $\mathrm{H}_{862 / 921}$ induced mild lesion (BLS 1.5); $\mathrm{H}_{921 / 783}$ hardly induce lesion (BLS 0). As to the ration of bursal weight and body weight, rHarbin- 1 and $\mathrm{H}_{783 / 862}$ showed severe bursal atrophy $(3.39,3.94$ respectively). There was no remarkable difference among $\mathrm{H}_{253 / 284}, \mathrm{H}_{862 / 921}, \mathrm{H}_{921 / 783}$ and negative control (4.0, 4.8, 4.94, 4.71 respectively) (Table 3). The above-mentioned results demonstrated that V862 and I921 in VP3 are probably the major virulence determinants, furthermore, 862 and 921 in VP3 has the stronger ability to manage virulence than 253 and 284 in VP2.

\section{DISCUSSION}

In recent years, many investigators have shown that mutations in the viral genome often lead to changes in the virulence, pathogenesis of animal viruses. A single amino acid substitution in the West Nile Virus Nonstructural protein NS2A disables its ability to inhibit Alpha/Beta interferon induction and attenuates virus in mice [18]; point mutations in an infectious bovine viral diarrhoea virus type 2 cDNA transcript yields an attenu- 
Table 3. Results of chicken challenged by four recombinant viruses.

\begin{tabular}{|c|c|c|c|c|c|}
\hline Virus & $\begin{array}{l}\text { Number of } \\
\text { Chickens }^{*}\end{array}$ & Mortality & $\begin{array}{l}\text { Avg bursa/body wt, } \\
\text { Ratio (SD), } 10^{3}\end{array}$ & $\begin{array}{l}\text { Avg BLS } \\
\text { (SD) }\end{array}$ & Pathological Lesions \\
\hline $\mathrm{H}_{253 / 284}$ & 8 & $1 / 8$ & $4.0(2.0)^{\mathrm{ab}}$ & $1.6(1.1)^{b}$ & individual lymphatic nodule necrosis and atrophy in dead chicken \\
\hline $\mathrm{H}_{783 / 862}$ & 8 & $5 / 8$ & $3.9(1.5)^{\mathrm{ab}}$ & $3.1(2.6)^{\mathrm{c}}$ & lymphatic nodule severe necrosis and atrophy in dead chickens \\
\hline $\mathrm{H}_{862 / 921}$ & 8 & $1 / 8$ & $4.8(1.4)^{\mathrm{b}}$ & $1.5(1.4)^{\mathrm{b}}$ & lymphatic nodule partly necrosis and slightly atrophy in dead chicken \\
\hline $\mathrm{H}_{921 / 783}$ & 8 & $0 / 8$ & $4.9(0.9)^{\mathrm{b}}$ & $0(0.0)^{\mathrm{a}}$ & $\begin{array}{l}\text { lymphatic nodule slightly atrophy and widen interstice close to } \\
\text { normal }\end{array}$ \\
\hline rHarbin-1 & 8 & $7 / 8$ & $3.4(0.9)^{\mathrm{a}}$ & $5(0.0)^{\mathrm{d}}$ & lymphatic nodule appear necrosis, congest and hemorrhage \\
\hline $\begin{array}{l}\text { Native } \\
\text { Harbin-1 }\end{array}$ & 8 & $7 / 8$ & $3.1(0.4)^{\mathrm{a}}$ & $5(0.0)^{\mathrm{d}}$ & $\begin{array}{l}\text { lymphatic nodule appear necrosis, congest and severe hemorrhage, } \\
\text { atrophy }\end{array}$ \\
\hline $\begin{array}{l}\text { Negative } \\
\text { control }\end{array}$ & 8 & $0 / 8$ & $4.7(0.3)^{b}$ & $0(0.0)^{\mathrm{a}}$ & normal \\
\hline
\end{tabular}

${ }^{*}$ The indicated number of 4-week-old SPF chickens were infected via the eye and nose drop; ${ }^{* *}$ BLS of BF of each chicken investigated. Values within the same row with the same superscript letters are not significant $(P<0.05)$.

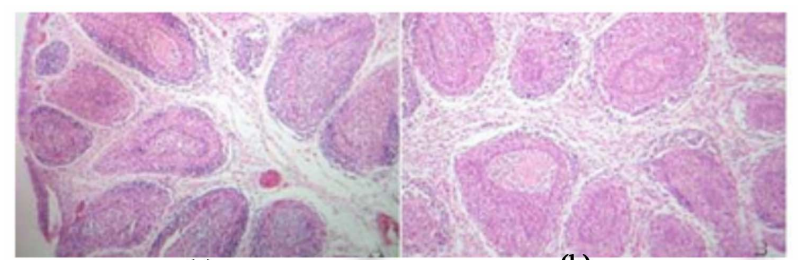

(a)

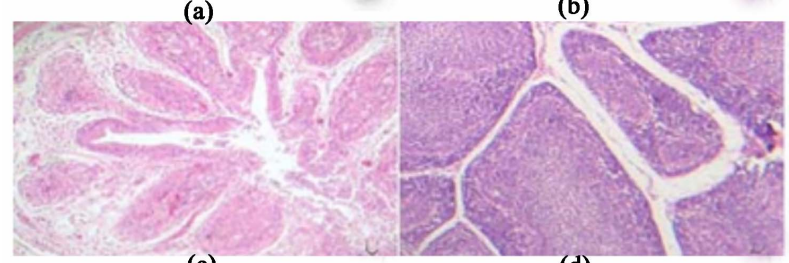

(c)

(d)
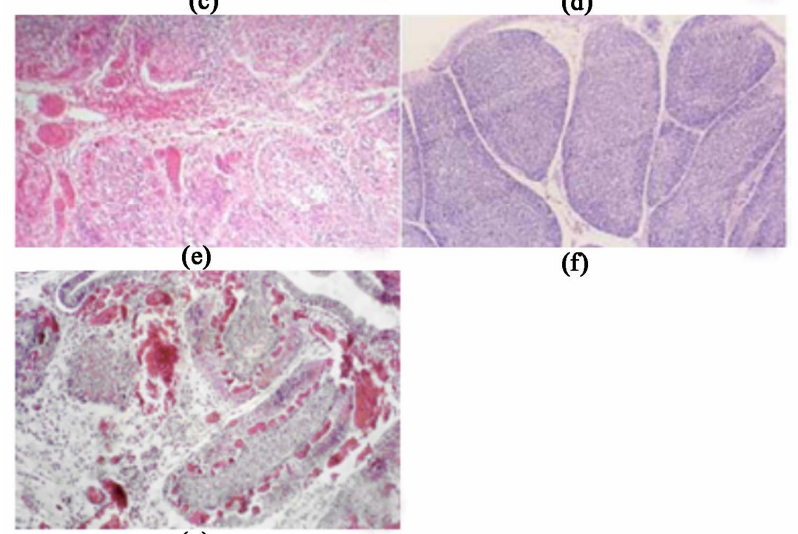

(g)

Figure 1. Microscopic pathological effect in bursae challenged by virus mutants $(10 \times 20)\left(\right.$ a) $\mathrm{H}_{253 / 284}$ single lymph nodule necrosis, atrophy, BLS 4; (b) $\mathrm{H}_{783 / 862}$ lymph nodule severe necrosis, atrophy, BLS 4.5; (c) $\mathrm{H}_{862 / 921}$ lymph nodule partly necrotize, BLS 2.3; (d) $\mathrm{H}_{921 / 783}$ close to normal, BLS 0; (e) rHarbin-1 lymph nodule necrosis, congest and hemorrhage BLS 5; (f) CK (negative); (g) Harbin-1 (positive control) lymph nodule necrosis, congest and severe hemorrhage, atrophy BLS 5. ated and protective viral progeny. Virulence of swine vesicular disease virus is determined at two amino acids in capsid protein VP1 and 2A protease [14]. Above mentioned phenomena elicit researchers on IBDV and they dedicated to study the virulence mechanism. A number of researchers such as Brandt, Yamaguchi, Lim, Mundt and so forth assumed position 253, 279, 284 amino acids in VP2 hypervarible region control phenotype, and could bind with B lymphocyte [3,17,21,29]. Lots of evidence showed that hypervarible region in VP2 involved in conformation dependent epitope and stimulate the chicken to produce protective neutralizing antibody $[10$, 29].

The result of chickens challenged with viruses showed that $\mathrm{H}_{253 / 284}$ could induced slighter lesion than parental virus vvIBDV (Harbin-1), but in $\mathrm{H}_{783 / 862}$ group, there are two kinds of appearance ,the bursa in alive chickens had not showed pathological sign, which could be due to the individual difference, but the bursae of dead chickens showed severe necrosis and atrophy, B lymphocyte depletion was up to the same $80-90 \%$ as Harbin-1; in $\mathrm{H}_{862 / 921}$ group, a bursa of dead chicken had the same pathological lesion as Harbin-1, lymphocyte depletion up to above $90 \%$, in other bursae of alive chickens depletion is only $10-20 \%$, and appear partly necrosis and atrophy; in $\mathrm{H}_{921 / 783}$ group bursae had very slightly pathological lesion except minor widening interstice, suggesting bursa was slight swollen. Therefore, $\mathrm{H}_{921 / 783}$ virus appeared the slightest pathological lesion among all virus mutants. Compared with mDT-VP3C and mDCT-VP3C rescued by Boot who substituted the Cterminal part of VP3 of serotype 1 vvIBDV (isolate D6948) for the corresponding part of serotype 2 IBDV [22], $\mathrm{H}_{921 / 783}$ induced slighter pathological lesion than 
mDT-VP3C and mDCT-VP3C. mDT-VP3C and mDCTVP3C could induced same bursa lesion as wild type D6948 and rD6948, suggesting mDT-VP3C and mDCTVP3C had stronger residential virulence, but $\mathrm{H}_{921 / 783}$ virus hardly has no residential virulence.

Our experiment demonstrated that VP3 and VP2 contain the determinant for virulence too besides VP2 in one strain. However, up to now most researches assume VP2 play an important role in virulence. The reason for this paradox about virulence controlling mechanism is unknown. Molecular determinant of virulence may depend the strains used. In addition we used two alterations of amino acid in this paper. Single alterations of aa 783 , 862 and 921 were not tested, further study may be necessary to identify if single amino acid function or both of them function in virulence at the same time.

\section{CONCLUSIONS}

V862, I921 in VP3 is obvious virulence marker however I921 has more potential ability to control virulence than V862 and H783. Through animal challenge test we make clear the site in VP2 and VP3 involved in virulence, furthermore, the ability of 862 and 921 to control virulence in VP3 is more powerful than 253 and 284 in VP2.

\section{ACKNOWLEDGEMENTS}

We thank Professor Zhizhong Cui in Shandong Agricultural University for his assistance in animal experiment. Professor Zhao Deming in National Animal TSE Lab in China Agricultural University is gratefully acknowledged for his assistance in Quantitative realtime PCR experiment. This study was supported by Chinese NSFC grant No. 9893290 and INCO-China grant ERBIC18CT98-0330.

\section{REFERENCES}

[1] Cosgrove, A.S. (1962) An apparently new disease of chickens: Avian nephrosis. Avian Diseases, 6(3), 385389.

[2] Wei, Y.W., Yu, X.P., Zheng, J.T., Chu, W.Y., Xu, H., $\mathrm{Yu}, \mathrm{X} . \mathrm{M}$. and Yu, L. (2008) Reassortant infectious bursal disease virus isolated in China. Virus Research, 131(2), 279-282.

[3] Delmas, B. (2005) Birnaviridae. In: Virus Taxonomy, 8th Report of the International Committee on Tax-onomy of Viruses, Fauquet, C.M., Mayo, M.A., Maniloff, J., Desselberger, U. and Ball, L.A., Eds, Elsevier Academic, London, 561-569.

[4] Müller, H. (2003) Research on infectious bursal diseasethe past, the present and the future. Veterinary Microbiology, 97(1-2), 153-165.
[5] Yamaguchi, T., Ogawa, M., Miyoshi, M., Inoshima, Y., Fukushi, H. and Hirai, K. (1997) Sequence and phylogenetic analyses of highly virulent infectious bursal disease virus. Archives of Virology, 142(7), 1441-1458.

[6] Ikuta, N., El-Attrache, J., Villegas, P., Garcia, E.M., Lunge, V.R., Fonseca, A.S., Oliveira, C. and Marques, E.K. (2001) Molecular characterization of Brazilian infectious bursal disease viruses. Avian Diseases, 45(2), 297-306.

[7] Dobos, P., Hill, B.J., Hallett, R., Kells, D.T.C., Becht, H. and Teninges, D. (1979) Biophysical and biochemical characterization of five animal viruses with bisegmented double-stranded RNA genomes. Journal of Virology, 32(2), 593-605.

[8] Kibenge, F.S., Qian, B., Cleghorn, J.R. and Martin, C.K. (1997) Infectious bursal disease virus polyprotein processing does not involve cellular proteases. Archives of Virology, 142(12), 2401-2419.

[9] Spies, U., Müller, H. and Becht, H. (1987) Properties of RNA polymerase activity associated with infectious bursal disease virus and characterization of its reaction products. Virus Research, 8(2), 127-140.

[10] Von Einem, U.I., Gorbalenya, A.E., Schirrmeier, H., Behrens, S.E., Letzel, T. and Mundt, E. (2004) VP1 of infectious bursal disease virus is an RNA-dependent RNA polymerase. Journal of General Virology, 85(8), 2221-2229.

[11] Dobos, P. (1993) In vitro guanylylation of infectious pancreatic necrosis virus polypeptide VP1. Virology, 193(1), 403-413.

[12] Spies, U. and Müller, H. (1990) Demonstration of enzyme activities requiredfor cap structure formation in infectious bursal disease virus, a member of the birnavirus group. Journal of General Virology, 71(Pt4), 977-981.

[13] Brown, M.D., Green, P. and Skinner, M.A. (1994) VP2 sequences of recent European 'very virulent' isolates of infectious bursal disease virus are closely related to each other but are distinct from those of 'classical' strains. Journal of General Virology, 75(Pt3), 675-680.

[14] Fahey, K.J., Erny, K. and Crooks, J. (1989) A conformational immunogen on VP2 of infectious bursal disease virus that induces virus-neutralizing antibodies that passively protect chickens. Journal of General Virology, 70(Pt6), 1473-1481.

[15] Letzel, T., Coulibaly, F., Rey, F.A., Delmas, B., Jagt, E.W., van Loon, A.A.M. and Mundt, E. (2007) Molecular and structural bases for the antigenicity of VP2 of infectious bursal disease virus. Journal of Virology, 81(23), 12827-12835.

[16] Yamaguchi, T., Ogawa, M., Inoshima, Y., Miyoshi, M., Fukushi, H. and Hirai, K. (1996) Identification of sequence changes responsible for the attenuation of highly virulent infectious bursal disease virus. Virology, 223(1), 219-223.

[17] Mundt, E. (1999) Tissue culture infectivity of different strains of infectious bursal disease virus is determined by distinct amino acids in VP2. Journal of General Virology, 
80(8), 2067-2076.

[18] Jackwood, D.J., Sreedevi, B., Lefever, L.J. and SommerWagner, S.E. (2008) Studies on naturally occurring infectious bursal disease viruses suggest that a single amino acid substitution at position 253 in VP2 increases pathogenicity. Virology, 377(1), 110-116.

[19] Brandt, M., Yao, K., Liu, M., Heckert, R.A. and Vakharia, V.N. (2001) Molecular determinants of virulence, cell tropism, and pathogenic phenotype of infectious bursal disease virus. Journal of Virology, 75(24), 1197411982.

[20] Van Loon, A.A.W.M., de Haas, N., Zeyda, I. and Mundt, E. (2002) Alteration of amino acids in VP2 of very virulent infectious bursal disease virus results in tissue culture adaptation and attenuation in chickens. Journal of General Virology, 83(1), 121-129.

[21] Boot, H.J., ter Huurne, A.A., Hoekman, A.J., Peeters, B.P. and Gielkens, A.L. (2000) Rescue of very virulent and mosaic infectious bursal disease virus from cloned cDNA: VP2 is not the sole determinant of the very virulent phenotype. Journal of Virology, 74(15), 6701-6711.

[22] Boot, H.J., ter Huurne, A.A., Hoekman, A.J., Pol, J.M, Gielkens, A.L. and Peeters, B.P. (2002) Exchange of the C-terminal part of VP3 from very virulent infectious bursal disease virus results in an attenuated virus with a unique antigenic structure. Journal of Virology, 76(20), 10346-10355.

[23] Yamaguchi, T., Kondo, T., Inoshima, Y., Ogawa, M., Miyoshi, M., Yanai, T., Masegi, T., Fukushi, H. and
Hirai, K. (1996) In vitro attenuation of highly virulent infectious bursal disease virus: Some characteristics of attenuated strains. Avian Diseases, 40(3), 501-509.

[24] Mundt, E. and Vakharia, V.N. (1996) Synthetic transcripts of double-stranded Birnavirus genome are infectious. Proceedings of National Academy of Sciences of USA, 93(11-12), 11131-11136.

[25] Nunoya, T., Tajima, M. and Itakura, C. (1991) Primary culture of chicken bursal plical epithelium. Research in Veterinary Science, 50(3), 352-354.

[26] Eterradossi, N., Toquin, D., Rivallan, G. and Guittet, M. (1997) Modified activity of a VP2-located neutralizing epitope on various vaccine, pathogenic and hypervirulent strains of infectious bursal disease virus. Archives of Virology, 142(2), 255-270.

[27] Synder, D.B., Lana, D.P., Cho, B.R. and Marquardt, W.W. (1988) Group and strain-specific neutralization sites of infectious bursal disease virus defined with monoclonal antibodies. Avian Diseases, 32(5), 527-534.

[28] Hierholzer, J.C. and Killington, R.A. (1996) Suspension assay method. Virology Methods Manual, Academic, San Diego, 39-40.

[29] Schröder, A., van Loon, A.A.W.M., Goovaerts, D. and Mundt, E. (2000) Chimeras in noncoding regions between serotypes I and II of segment A of infectious bursal disease virus are viable and show pathogenic phenotype in chickens. Journal of General Virology, 81(23), 533-540. 